05,15

\title{
Самоорганизация в системах магнитных анизотропных наночастиц
}

\author{
(C) А.В. Гудкова, Е.C. Пьянзина \\ Уральский федеральный университет им. Б.Н. Ельцина, \\ Екатеринбург, Россия \\ E-mail: annagudkova94@gmail.com
}

\begin{abstract}
В данной работе представлено комплексное исследование систем магнитных анизотропных наночастиц с помощью компьютерного эксперимента в широком диапазоне параметров системы. Проведен кластерный анализ, рассчитаны различные средние характеристики образующихся кластеров, вычислены начальная магнитная восприимчивость и радиальная функция распределения. С помощью изменения характеристик наночастиц (их формы, величины магнитного момента) можно изменить макроскопический отклик системы, что воплощает идею усовершенствования и разработки новых материалов с контролируемыми свойствами.
\end{abstract}

Работа выполнена при финансовой поддержке Министерства образования и науки (проект № 3.1438.2017/ПЧ).

DOI: $10.21883 /$ FTT.2017.11.45054.11k

\section{1. Введение}

В последние годы исследования систем магнитных анизотропных наночастиц привлекают большое внимание тех, кто занимается изучением магнитных жидкостей. Подобные системы, содержащие новый тип частиц, представляют особый интерес благодаря способности образовывать различные жидкокристаллические фазы, такие как нематическая, смектическая и др. [1-4]. Центральное место в этих исследованиях занято системами жестких/мягких эллипсоидов или сфероцилиндров, в центрах масс которых расположены точечные дипольные моменты, сонаправленные с главными осями. Подобные частицы имеют интересную особенность: с изменением формы меняется структура их основного состояния - конфигурация магнитных моментов „голова-хвост“ становится энергетически менее выгодной, чем у антипараллельной пары диполей [5]. Такой структурный переход в основных состояниях приводит также и к изменениям в микроструктуре систем анизотропных наночастиц, когда тепловая энергия становится сравнимой с энергией межчастичного взаимодействия. Ориентация дипольного момента относительно осей частицы также влияет на поведение системы частиц в целом. В данной работе рассматриваются эллипсоидальные частицы, магнитный момент которых направлен вдоль главной оси вращения. Для таких систем проводится детальный анализ межчастичных корреляций, макроскопических свойств системы в широком диапазоне параметров, чтобы выявить возможные структурные переходы и оценить возможность управления макроскопическим откликом системы, что воплощает идею настройки и разработки новых материалов с контролируемыми свойствами.

\section{2. Магнитные эллипсоиды}

Рассматриваемая система состоит из магнитных анизотропных наночастиц, представляющих собой эллип- соиды вращения. Соотношение полуосей задается параметром $X_{0}=b / a$, который показывает, насколько форма эллипсоидальной частицы отличается от сферической. В данной работе были рассмотрены наночастицы со значениями параметра $X_{0}=1.1,1.4,1.7,2.0,2.3$. Общий потенциал взаимодействия таких частиц складывается из двух: стерического и магнитного диполь-дипольного.

Для описания магнитного взаимодействия был использован потенциал $U_{d}$ :

$$
U_{d}(i, j)=-\left[3\left(\mathbf{m}_{i}, \mathbf{r}_{i j}\right) / r_{i j}^{5}-\left(\mathbf{m}_{i}, \mathbf{m}_{j}\right) / r_{i j}^{3}\right],
$$

где $\mathbf{r}_{i j}$ - радиус-вектор двух частиц, $\mathbf{m}_{i(j)}$ - магнитный момент частицы $i(j)$.

Для описания стерического взаимодействия анизотропных частиц использовали модифицированный потенциал Гей-Берне (2) [6,7], который широко применяется для исследования несферических частиц. Этот потенциал учитывает не только расстояние между центрами частиц, но и их ориентацию.

$$
U_{\mathrm{GB}}=\left\{4 \varepsilon\left[A^{12}-A^{6}\right]+\varepsilon, r_{i j} \leq r_{c}, 0, r_{i j}>r_{c},\right.
$$

где

$$
\begin{gathered}
A=A\left(\mathbf{u}_{i}, \mathbf{u}_{j}, \mathbf{R}_{i j}\right)=\sigma_{0} /\left[r_{i j}-\sigma\left(\mathbf{u}_{i}, \mathbf{u}_{j}, \mathbf{R}_{i j}\right)+\sigma_{0}\right] \\
\sigma\left(\mathbf{u}_{i}, \mathbf{u}_{j}, \mathbf{R}_{i j}\right)=\sigma_{0}\left[1-\varphi / 2\left(\mathbf{R}_{i j} \mathbf{u}_{i}+\mathbf{r}_{i j} \mathbf{u}_{j}\right)^{2} /\left(1+\varphi \mathbf{u}_{i} \mathbf{u}_{j}\right)\right. \\
\left.+\left(\mathbf{R}_{i j} \mathbf{u}_{i}-\mathbf{r}_{i j} \mathbf{u}_{j}\right)^{2} /\left(1-\varphi \mathbf{u}_{i} \mathbf{u}_{j}\right)\right]^{-1 / 2} \\
\varepsilon \equiv \varepsilon\left(\mathbf{u}_{i}, \mathbf{u}_{j}\right)=\varepsilon_{0}\left[1-\varphi^{2}\left(\mathbf{u}_{i} \mathbf{u}_{j}\right)^{2}\right]^{-1 / 2} \\
\varphi=\left[X_{0}^{2}-1\right]\left[X_{0}^{2}+1\right]
\end{gathered}
$$

В данном случае за вектор $\mathbf{R}_{i j}=\mathbf{r}_{i j} / r_{i j}=\left(\mathbf{r}_{i}-\mathbf{r}_{j}\right) / r_{i j}$ принимается единичный вектор, коллинеарный вектоpy, соединяющему центры частиц, а $\mathbf{u}_{i(j)}-$ единичный вектор, направленный вдоль главной оси, $r_{c}=\left(2^{1 / 6}-1\right) \sigma_{0}+\sigma\left(\mathbf{u}_{i}, \mathbf{u}_{j}, \mathbf{R}_{i j}\right)-$ критический радиус. Параметр $\sigma_{0}$ принимается равным $\sqrt{2} a$. 
Средние характеристики кластеров при $\rho=0.08$

\begin{tabular}{l|c|c|c|c}
\hline \multicolumn{2}{c|}{ Соотношение полуосей } & \multicolumn{2}{|c}{1.1} & \multicolumn{2}{c}{2.3} \\
\multicolumn{1}{c|}{ эллипсоида $X_{0}$} & 1 & 5 & 1 & 5 \\
Магнитный момент $\mathbf{m}^{2}$ & 2.126 & 3.647 & 2.02 & 2.072 \\
Средняя длина кластера $L$ & 14.718 & 71.891 & 3.441 & 12.141 \\
Степень агрегированности $P$ & Взаимная ориентация & „г конфигураций & $89 \%$ конфигураций \\
диполей в кластере & „глова-хвост“ & „голова-хвост“ & п5\% антипараллельных & $99 \%$ антипараллельных \\
пар
\end{tabular}

Для проведения детального анализа поведения системы магнитных анизотропных частиц в широком диапазоне параметров были проведены компьютерные эксперименты методом молекулярной динамики в среде ESPResSo [8]. В данных экспериментах используются безразмерные параметры: все величины, связанные с длиной, нормируются на значение короткой оси эллипсоида (она все время равна 1). Безразмерная температура $T^{*}=k T / \varepsilon_{0}=1$, где $k T-$ тепловая энергия, $\varepsilon_{0}$ - энергетический параметр. Безразмерный магнитный момент $m$ измеряется в единицах $1 / \sqrt{T^{*}}$ и расположен в центре частицы. Была рассмотрена система, состоящая из 512 частиц, с периодическими граничными условиями; для расчета дальнодействующего магнитного диполь-дипольного взаимодействия использовали алгоритм $\mathrm{P}^{3} \mathrm{M}$ [8]. Для достижения равновесного состояния сначала был проведен начальный расчет с периодом $10^{5} \Delta T^{*}$, после чего было начато исследование микроструктуры и макроскопических характеристик системы. Для частиц различной формы проводили исследование при одинаковой числовой концентрации $\rho$ в широком диапазоне значений. Использование безразмерных параметров позволяет изучать различные наночастицы, обладающие схожими свойствами. Примерами таких систем могут служить сферические магнитные наночастицы из магнетита (с намагниченностью насыщения $480 \mathrm{kA} / \mathrm{m}$ ), внедренные в эллипсоидальные немагнитные мягкие наночастицы. Для таких частиц безразмерный магнитный момент $\mathbf{m}^{2}=5$ при комнатной температуре соответствует частице с диаметром $\sim 15 \mathrm{~nm}$, а момент $\mathbf{m}^{2}=3-$ диаметру $12.5 \mathrm{~nm}$. Также могут быть рассмотрены никелевые наноиглы (намагниченность насыщения материала $488 \mathrm{kA} / \mathrm{m}$ ), покрытые немагнитной оболочкой, с диаметром в центральном сечении $\sim 50 \mathrm{~nm}$ и различной длиной (например, для $X_{0}=2.3$ равной $115 \mathrm{~nm}$ ).

\section{3. Микроструктура системы}

Полученные в результате компьютерных экспериментов большие массивы данных, содержащие информацию о координатах частиц и ориентации их диполей, стали основой для проведения кластерного анализа, который позволяет определить средние характеристики образовавшихся в системе агрегатов. Для этого была использована теория графов: каждая частица становится вершиной графа, а связи между ними задаются по следующему критерию: энергия магнитного диполь-дипольного взаимодействия (1) должна быть отрицательна, а межчастичное расстояние не должно превосходить критическое. Были рассчитаны средняя длина кластера (количество частиц в нем), степень агрегированности системы (какой процент частиц находится в кластерах), изучена взаимная ориентация магнитных моментов.

В таблице показаны средние характеристики системы в зависимости от величины магнитного момента и
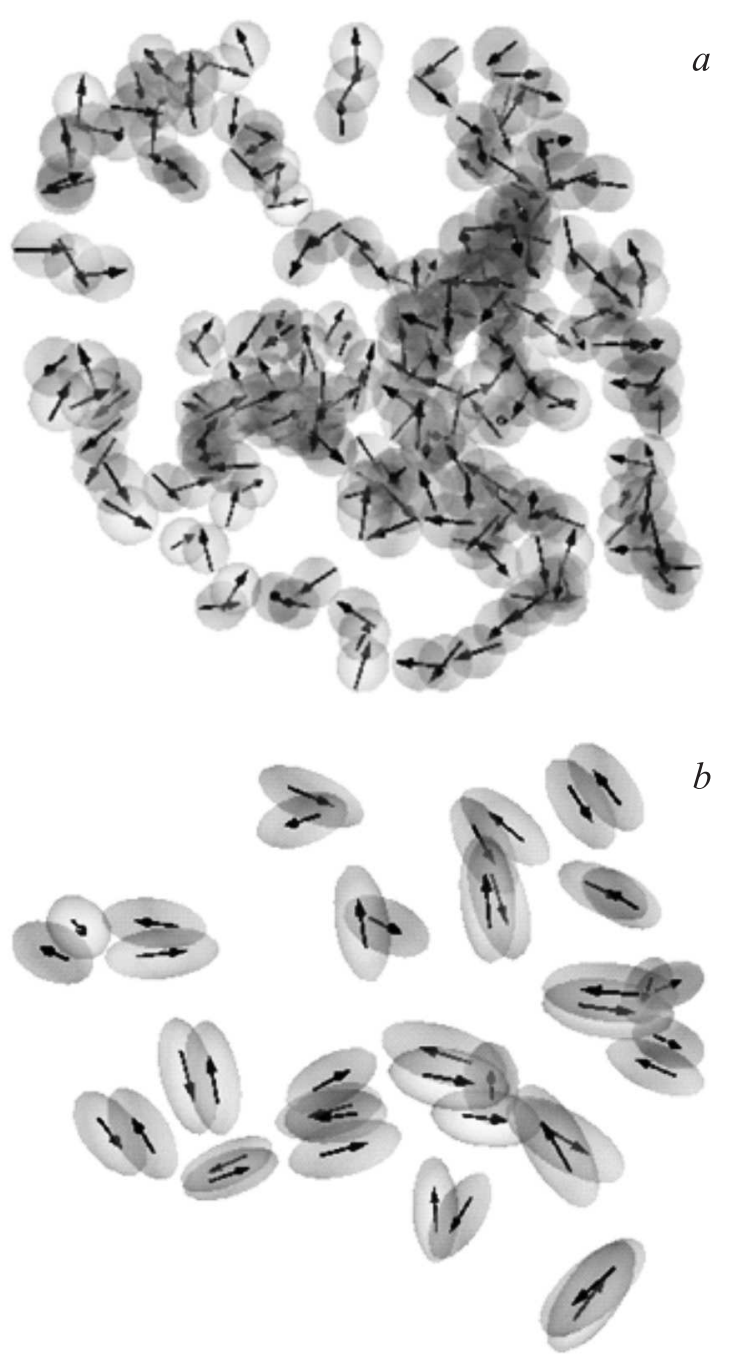

Рис. 1. Наглядное представление кластеров системы с концентрацией $\rho=0.08$, параметрами $\mathbf{m}^{2}=5$ и $X_{0}=1.1(a), 2.3(b)$. 
формы наночастицы. Для данного примера была выбрана концентрация $\rho=0.08$. Однако такое же качественное поведение наблюдается для систем при всех других концентрациях, различаются лишь количественные значения.

Проведенный анализ показал, что наночастицы, форма которых близка к сферической, в отличие от вытянутых эллипсоидальных частиц, демонстрируют высокую степень самоорганизации, причем возрастает не только количество кластеров в системе, но и среднее количество частиц в кластере. Увеличение магнитного момента в случае сферических частиц приводит к увеличению количества агрегатов в системе, а также к тому, что магнитные моменты частиц становятся практически сонаправленными, что повышает отклик такого кластера на внешнее магнитное поле. Если частицы вытянуты, с ростом величины магнитного момента взаимная ориентация диполей становится такой, что конфигурации „голова-хвост“ практически не встречаются: при различных концентрациях количество антипараллельных пар возрастает с 95\% до 99\%. Таким образом, магнитный момент оказывает меньшее влияние на поведение системы эллипсоидальных частиц.

Наглядное представление полученных кластеров без одиночных частиц приведено на рис. $1, a, b$.

Таким образом, можно сделать вывод о том, что форма частиц влияет на характеристики образующихся в процессе самоорганизации кластеров.

\section{4. Макроскопический отклик системы}

После изучения микроструктуры системы были рассмотрены ее макроскопические свойства. Начальная магнитная восприимчивость является одной из самых важных характеристик магнитных мягких материалов, поскольку показывает, насколько сильны корреляции дипольных моментов в системе и насколько чувствительна система к внешнему бесконечно малому магнитному полю.

Графики зависимости начальной магнитной восприимчивости от величины магнитного момента частицы представлены на рис. 2, $a-c$.

На основании приведенных графиков можно сделать вывод о том, что начальная восприимчивость возрастает при увеличении концентрации частиц и при возрастании магнитного момента. Начальная восприимчивость частиц сферической формы возрастает быстрее и достигает бо́льших значений, чем в случае эллипсоидальных частиц. Это вызвано тем, что в системе образуются цепочки, которые скоррелированно реагируют на внешнее поле. С ростом анизотропии формы частиц уменьшается не только абсолютное значение восприимчивости, но и его относительное изменение становится меньше. Общее снижение межчастичных дипольных корреляций и наблюдаемого магнитного отклика может быть объяснено изменением в типе самоорганизации частиц, что
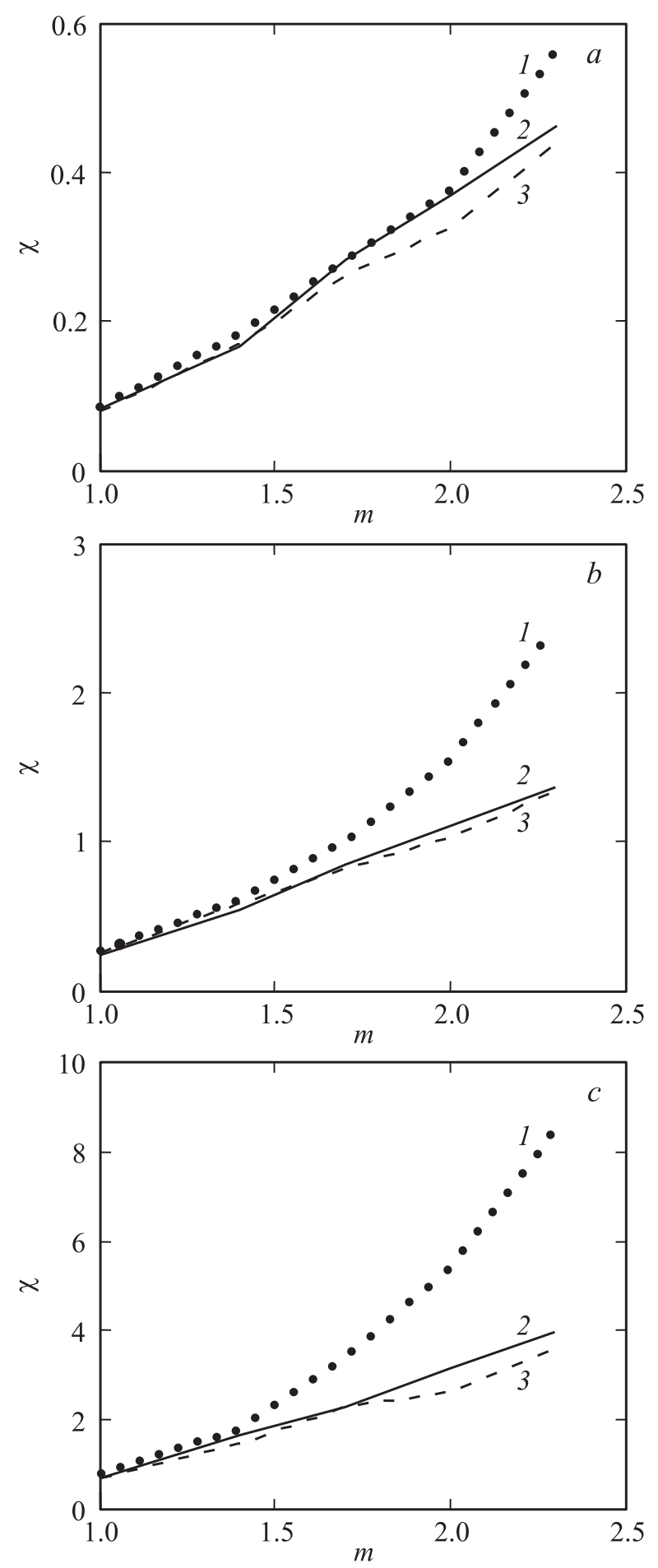

Рис. 2. Зависимость начальной магнитной восприимчивости от величины магнитного момента наночастиц с концентрацией $\rho=0.02(a), 0.06(b), 0.16(c) ; X_{0}=1.1(1), 1.7(2), 2.3(3)$.

вызвано сменой конфигурации основного состояния системы. Так как энтропия антипараллельной пары достаточно мала, то даже при больших значениях магнитного момента много таких пар не образуется. Кроме того, магнитный момент данной структуры в среднем равен 

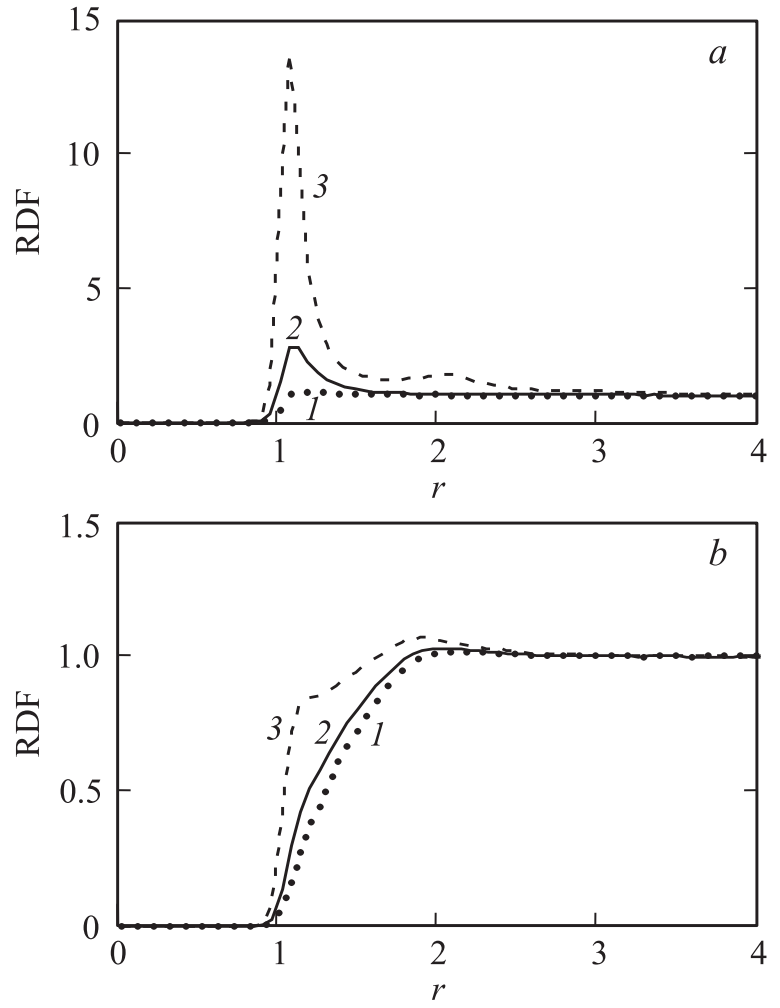

Рис. 3. Радиальная функция распределения как функция расстояния между центрами частиц, рассмотренная при концентрации $\rho=0.16$, параметр $X_{0}=1.1(a), 2.3(b), \mathbf{m}^{2}=1(1)$, $3(2), 5(3)$.

нулю, поэтому восприимчивость такого объекта ниже, чем дублета с ориентацией „голова-хвост“.

Также различное поведение наночастиц различной формы наблюдается и при рассмотрении радиальной функции распределения (RDF) - плотности вероятности найти пару частиц на заданном расстоянии (рис. 3).

Как можно заметить, поведение радиальной функции распределения частиц эллипсоидальной формы практически не меняется в зависимости от различных значений магнитных моментов. Можно сказать, что стерическое взаимодействие может экранировать магнитное. В случае частиц, близких к сферическим, с ростом величины магнитного момента возрастает первый пик радиальной функции распределения, что говорит об образовании кластеров в системе. Таким образом, форма частицы может достаточно сильно изменить макроскопический отклик всей системы.

\section{5. Заключение}

В настоящей работе было проведено исследование самоорганизации в системах магнитных анизотропных частиц, а также влияния данного процесса на поведение системы при комнатной температуре. Для этого были использованы компьютерные эксперименты, проведенные методом молекулярной динамики. В широком диапазоне изменения величины магнитного момента и концентрации частиц образование кластеров становится менее выраженным, если анизотропия формы частиц растет. Из того, что антипараллельные пары становятся энергетически более выгодными, чем конфигурация „голова-хвост“, следует, что при комнатной температуре происходит образование нестабильных пар даже при большом значении дипольного момента. Кроме того, общий магнитный момент антипараллельной пары мал, а значит, восприимчивость такого объекта значительно ниже, чем объекта с конфигурацией „голова-хвост“. Следовательно, форма анизотропии существенно влияет на микроструктуру системы магнитных наночастиц.

Таким образом, при использовании анизотропии формы частиц в качестве управляющего параметра можно изменять самоорганизацию в системе от цепочек с ориентацией магнитных моментов „голова-хвост“ (почти сферические частицы) до пространственно однородной системы (эллипсоидальные частицы), не изменяя при этом значение намагниченности насыщения. Это может оказаться важным для применения в медицине фактором, когда сильный магнитный отклик наночастиц должен сочетаться с отсутствием значительной агрегации частиц. Таким образом, форма наночастиц может быть эффективно использована в качестве управляющего параметра для микроструктуры системы, а сами системы будут полностью воплощать идею настройки и разработки новых материалов с контролируемыми свойствами.

\section{Список литературы}

[1] A.J. Arduengo III, H.V.R. Dias, R.L. Harlow, M. Kline. J. Am. Chem. Soc., 114, 5530 (1992).

[2] A. Abarca, P. Gómez-Sal, A. Martín, M. Mena, J.M. Poblet, C. Yélamos. Inorg. Chem. 39, 642 (2000).

[3] F. Chami, M.R. Wilson. J. Am. Chem. Soc. 132, 7794 (2010).

[4] M. Nakade, T. Ikeda, M. Ogawa. J. Mater. Sci. 42, 4815 (2007).

[5] S. Kantorovich, E. Pyanzina, F. Sciortino. Soft Matter 9, 6594 (2013).

[6] J.G. Gay, B.J. Berne. J. Chem. Phys. 74, 3316 (1981).

[7] S. Kantorovich, E. Pyanzina, C. De Michele, F. Sciortino. Soft. Matter 9, 4412 (2013).

[8] A. Arnold, B.A. Mann, H. Limbach, C. Holm. Gesellschaft für wissenschaftliche Datenverarbeitung mbh 63, 43 (2004). 